CORRIGENDUM

\title{
A Winogradsky-based culture system shows an association between microbial fermentation and cystic fibrosis exacerbation
}

Robert A Quinn, Katrine Whiteson, Yan-Wei Lim, Peter Salamon, Barbara Bailey, Simone Mienardi, Savannah E Sanchez, Don Blake, Doug Conrad and Forest Rohwer

The ISME Journal (2015) 9, 1052; doi:10.1038/ismej.2014.266

Correction to: The ISME Journal (2015) 9, 1024-1038; doi:10.1038/ismej.2014.234; published online 16 December 2014

Since the publication of this article, the authors have identified an error concerning the acknowledgements, namely that 'Cystic Fibrosis Research Inc.' was incorrectly listed as 'Cystic Fibrosis Research Foundation'.
The error has now been rectified, and the correct paper now appears in this issue. The html and online pdf versions have also been rectified, and now carry the correct paper.

The authors would like to apologise for any inconvenience this may have caused.

\section{ERRATUM}

\section{The host metabolite D-serine contributes to bacterial niche specificity through gene selection}

James PR Connolly, Robert J Goldstone, Karl Burgess, Richard J Cogdell, Scott A Beatson, Waldemar Vollmer, David GE Smith and Andrew J Roe

The ISME Journal (2015) 9, 1052; doi:10.1038/ismej.2015.17

Correction to: The ISME Journal (2015) 9, 1039-1051; doi: 10.1038/ismej.2014.242; published online 19 December 2014

Since the publication of this article, the publishers have identified that the sentence 'We found that D-serine alone caused repression of the Type 3 Secretion System (T3SS) and induced the SOS response is not an acronym' is incorrect and should be 'We found that D-serine alone caused repression of the Type 3 Secretion System (T3SS) and induced the SOS'.

The publishers would like to apologise for any inconvenience caused.

This error has now been rectified, and the corrected article appears in this issue. The html and online pdf versions have also been rectified, and now carry the corrected paper. 